\title{
Alemania y México: generación de valor más allá de los salarios
}

\author{
Tania E. González Alvarado \\ V. Ma. Antonieta Martin Granados
}

\section{Resumen}

El objetivo de este artículo es señalar que los programas de desempleo orientados a la protección del ingreso, mas no a la conservación del puesto de empleo, conducen a mayor precariedad laboral. Para alcanzar dicho objetivo se analizan dos contextos distintos: México y Alemania. El salario no es el único factor para elevar la calidad de vida de la población y contribuir al bienestar social.

\begin{abstract}
The aim of this paper is to show that unemployment benefits destroy jobs when the focus is the person. Unemployment benefits have had an adverse impact on job security. Two cases with different economic context are analyzed: Mexico and Germany. The salary is not the unique factor to improve life quality and social welfare.
\end{abstract}

Palabras clave: competitividad, valor económico, ingreso, empleo..

Keywords: competitiveness, economic value, income, employment.

\section{Introducción}

El paro que enfrentan los países desarrollados muestra la urgencia de generar mecanismos que contribuyan a la protección del empleo (puestos de trabajo) Sin embargo, los esfuerzos realizados en materia económica conllevan otros costos que, de ignorarse, pueden tener un efecto multiplicador negativo en la economía, conduciendo a que en el largo plazo la pérdida de puestos de empleo sea mayor a la esperada Esto ocurre cuando los mecanismos se orientan hacia el salario que la persona obtiene, en lugar de orientarse al puesto que la persona ocupa y complementarlos con otros factores no económicos que aumentan el bienestar 
La asociación aislada que se hace entre consumo e ingreso conduce a considerar que un salario alto dinamiza la economía a través del consumo exacerbado Sin embargo, la actual crisis global obliga a reorientar la lógica del sistema y considerar que un salario elevado no evita el sobreendeudamiento de las familias y el empobrecimiento; por el contrario, puede ser un incentivo para un mayor endeudamiento Toda vez que las familias caen en deuda de forma generalizada, se ralentiza la economía

Por otra parte, la evidencia demuestra que los programas de desempleo que se orientan hacia la protección del ingreso de la persona, mas no a la conservación del puesto de empleo, conducen a mayor precariedad laboral En este sentido el caso de Alemania, con la integración de la parte oriental, es significativo. Alemania, tras su reintegración, tuvo que generar nuevos mecanismos para enfrentar el paro laboral, toda vez que se reconoció que el seguro de desempleo sólo restaba competitividad a sus empresas En otras palabras, la protección para el desempleado, por sí sola, sólo conducía a la destrucción de los puestos de empleo, en lugar de conservarlos

Mediante el análisis del sector automotriz mexicano, complementado con el caso de Alemania como referencia, se coloca a discusión que no es el salario per se el que garantiza el bienestar, ni laprolongación del sistema capitalista Se requiere de estrategias que incidan en los mecanismos articuladores de los sistemas locales

\section{Estado Alemán como competidor de las empresas: un marco de referencia}

El salario no es el principal elemento que vincula a la empresa con la población, existen elementos sociales que se entremezclan en los sistemas económicos actuales y que mediante las actividades económicas pueden agotarse si no se procuran estrategias empresariales que permitan generar valor social en paralelo al valor económico (Martin y González, 2013)

El bienestar no se agota en el ingreso porque el ingreso se vincula al valor económico, pero el bienestar guarda mayor relación con el valor social que con el económico El bienestar subjetivo, la capacidad de tomar decisiones autónomas, la educación de calidad, la salud a lo largo del ciclo de vida, una comunidad segura y el empoderamiento ciudadano son tan importantes 
como el ingreso (Gray, 2015) Razón por la cual se requiere de estrategias complementarias ante programas de apoyo para quien queda desempleado, $\mathrm{y}$ para contrarrestar los efectos perversos de las transnacionales que recorren el mundo en busca de mano de obra barata

En ausencia de dichas estrategias, un salario elevado per se contribuirá a una mayor pobreza en el largo plazo, en lugar de mejorar el nivel de vida en la localidad La existencia de un grupo de personas contratado por una transnacional que de pronto tuviese la capacidad de comprar más ahora que antes, con un salario mucho mayor al que obtendría por realizar la misma tarea en una empresa local, podría derivar en mayor inflación. Esta situación haría que el poder adquisitivo de este grupo disminuyera en el mediano plazo, al mismo tiempo que empobrecería aún más a aquellos que mantuvieron los salarios locales Este cuadro pudiera presentarse con el establecimiento de una transnacional que pretende otorgar el mismo salario en un país rico que en un país pobre en aras de alcanzar la igualdad económica entre ambas regiones

Este planteamiento es con el fin de hacer notar que no son los bajos sala$\operatorname{rios}^{1}$ que las transnacionales promueven en la localidad destino lo que genera el efecto perverso, más bien es la forma como se articulan con la localidad La transnacional, al no considerar estrategias para la generación de valor social, conduce a exportar la riqueza del país pobre e importar la pobreza del rico, aumentando la desigualdad en una mayor proporción a la esperada, particularmente cuando la transnacional se mueve entre diferentes regiones con la intención de disminuir costos, siendo esta última la estrategia de competencia más usada en el nivel mundial ¿En qué coinciden los programas de desempleo con las estrategias salariales de las transnacionales? Que ambos se centran en la mayoría de los casos en el valor económico en términos del salario, olvidando que el valor social y sus mecanismos para generarlo son más importantes

Es aquí en donde encaja el caso de Alemania como referencia Para el Estado alemán la experiencia ha mostrado que la supresión de puestos de empleo suele tener lugar con mayor rapidez que la creación de nuevos puestos (Ministerio de Trabajo e Inmigración, 2009) De ahí la intervención del

1. Este documento no hace referencia a la sobreexplotación de los empleados por menos de un dólar diario, ni a las nuevas formas de esclavitud que imperan en el siglo xxi El término "salarios bajos" se usa para señalar que en comparación con los salarios ofrecidos por esa misma transnacional en sus países de origen, tienden a ser menores 
Estado en momentos de cambios estructurales a fin de proteger el empleo, siempre que de esta forma se conserven en el mercado laboral empleos económicamente rentables y sostenibles desde un punto de vista social y ecológico(Ministerio de Trabajoe Inmigración, 2009)

De acuerdoconel Instituto de Macroeconomía e Investigación Coyuntural (imk), la economía alemana está atravesando una profunda crisis (Ministerio de Trabajo e Inmigración, 2009) En Alemania existe un desaprovechamiento de parte de las capacidades industriales y un entorno desfavorable en las condiciones para el financiamiento a las empresas (Ministerio de Trabajo e Inmigración, 2009)

Una de las tendencias más relevantes es la compenetración de la industria y los servicios El valor añadido se deriva cada vez más de la cadena de valores en varios ramos y sectores, en lugar de generarse como en el pasado dentro de un sector más o menos aislado De esta forma, la industria actual consiste en una red compuesta por la producción industrial tradicional por una parte, y los servicios en el entorno de las industrias por otra (Ministerio de Trabajo e Inmigración, 2009) El sistema empresarial alemán está basado en redes complejas en las que es importante tanto la forma en que los agentes económicos se vinculan, como en su capacidad para generar valor social a través de dichos vínculos

A pesar de que la mayor parte del pib se genera en el sector servicios, éste depende sobre todo de la demanda de tales servicios en el entorno de las industrias que, a su vez, depende de la producción industrial y de la demanda final de bienes industriales (Ministerio de Trabajoe Inmigración, 2009).

La relevancia de un sector para el conjunto de la economía alemana no sólo se determina con base en el número de puestos de trabajo, sino que hay que tener en cuenta toda la cadena de valor (Ministerio de Trabajo e Inmigración, 2009)

El sector industrial es importante para la producción y el empleo en Alemania La europeización y globalización de la economía alemana y sus empresas ha afectado a las estructuras empresariales, los emplazamientos y los flujos de mercancías y capitales. De esta forma, la industria de automóviles, por ejemplo, ha creado redes transnacionales de producción En el ejemplo de Opel se puede observar cómo los vínculos internacionales se convierten en cargas sumamente complicadas (Ministerio de Trabajo e Inmigración, 2009) 
La elevación de las cifras de desempleo en Alemania en los últimos años ha ido en aumento, aunque de forma moderada Las empresas reducen la jornada de sus trabajadores para ganar tiempo (Ministerio de Trabajo e Inmigración, 2009)

De acuerdo con una encuesta realizada en 2007 por el Instituto wsi entre comités de empresa, gran parte de los empleadores en Alemania aprovecharon las medidas de reestructuración para deslocalizar el empleo (outsourcing), ejerciendo presión con este instrumento para conseguir reducciones salariales o ampliaciones de la jornada laboral (Ministerio de Trabajo e Inmigración, 2009)

El resultado fue un aumento en la jornada laboral de las empresas afectadas, se empeoró el clima empresarial y los puestos de trabajo fueron menos seguros que antes Para regiones que dependen tradicionalmente de un sector industrial determinado, los grandes procesos de reestructuración pueden tener fatales consecuencias en su infraestructura, a pesar de las subvenciones públicas (Ministeriode Trabajoe Inmigración, 2009) Como en otros Estados miembros de la ue, Alemania había apostado por el factor crecimiento, en el que se basaba la "estrategia de Lisboa", que justificaba la subvención de núcleos de crecimiento con la esperanza de que de ello también se beneficiaran las zonas periféricas (Ministerio de Trabajo e Inmigración, 2009)

Existe un sinfín de programas de fomento y subvenciones del Estado alemán y los länder (Estados federados que componen Alemania), cuyos rasgos principales se subdividen en cuatro categorías (Ministerio de Trabajo e Inmigración, 2009): fomento de las inversiones empresariales; fundación de empresas; fomento de la I+D; superación de periodos de crisis

En los últimos años el fomento a la economía se centra en el apoyo a innovaciones y subvenciones para las empresas pequeñas y medianas Cada año el Estado alemán y los länder prestan ayudas operacionales a empresas con un volumen de varios miles de millones de euros En 2007, la industria y el sector de servicios alemanes obtuvieron más de 14,000 millones de euros (según datos de la Comisión Europea de 2008), más que cualquier otro país europeo Si se relacionan estas subvenciones con el pib, Alemania ocupa el quinto lugar, después de Portugal, Suecia, Hungría y Malta (Ministerio de Trabajoe Inmigración, 2009)

Antes de la crisis, $9 \%$ de las empresas de los länder orientales y $4 \%$ de las de los occidentales obtenían subvenciones públicas para inversiones y para 
la adquisición de bienes, mientras que 13\% de las empresas del Este y $8 \%$ del Oeste de Alemania recibían subvenciones para cubrir gastos salariales De estas cuotas no se desprenden ni cifras concretas sobre el volumen de las ayudas pagadas, ni información sobre la cifra o la calidad de los puestos de trabajo creados o salvados con estos recursos Para obtener una subvención, las empresas tenían que demostrar que el objetivo de la inversión solicitada era el aumento de la competitividad y la creación de empleo Las empresas tenían que contribuir con recursos propios y demostrar que conservaban o creaban puestos de trabajo con su propio esfuerzo El número de los puestos de trabajo que se conservaban o creaban dependía del volumen de las inversiones (Ministerio de Trabajoe Inmigración, 2009)

Muchas veces, bajo estos programas, se mantiene una plantilla inferior a la realmente existente y se descuida por completo la calidad de los puestos prometidos Por ejemplo, las empresas no se comprometen a pagar salarios mínimos determinados, a facilitar ciertas condiciones de trabajo o a permitir la elección de un comité de empresa (Ministerio de Trabajoe Inmigración, 2009)

Cuadro 1

Alemania: indicadores de crecimiento, 2011-2015a

\begin{tabular}{|c|c|c|c|c|c|}
\hline Indicadores de crecimiento & 2011 & 2012 & 2013 & 2014 & $2015(e)$ \\
\hline pib (miles de millones de usd) & $3,631.44$ & $3,427.85$ & $3,635.96 \mathrm{e}$ & $3,820.46 \mathrm{e}$ & $3,908.80$ \\
\hline $\begin{array}{l}\text { pib (crecimiento anual en \%, } \\
\text { precio constante) }\end{array}$ & 3.4 & 0.9 & $0.5 \mathrm{e}$ & $1.4 \mathrm{e}$ & 1.5 \\
\hline pib per cápita (usd) & 45,208 & 42,569 & $44 \mathrm{e}$ & $47,201 \mathrm{e}$ & 48,226 \\
\hline $\begin{array}{l}\text { Saldo de la hacienda pública (en } \\
\% \text { del pib) }\end{array}$ & -1.2 & -0.1 & $0.6 \mathrm{e}$ & $0.5 e$ & 0.5 \\
\hline $\begin{array}{l}\text { Endeudamiento del Estado (en } \\
\% \text { del pib) }\end{array}$ & 80.0 & 81.0 & $78.4 \mathrm{e}$ & $75.5 \mathrm{e}$ & 72.5 \\
\hline Tasa de inflación (\%) & 2.5 & 2.1 & $1.6 \mathrm{e}$ & $0.9 \mathrm{e}$ & 1.2 \\
\hline $\begin{array}{l}\text { Tasa de paro (\% de la población } \\
\text { activa) }\end{array}$ & 6.0 & 5.5 & 5.3 & 5.3 & 5.3 \\
\hline $\begin{array}{l}\text { Balanza de transacciones } \\
\text { corrientes (miles de millones de } \\
\text { usd) }\end{array}$ & 228.15 & 252.28 & $254.93 e$ & $236.99 e$ & 228.39 \\
\hline $\begin{array}{l}\text { Balanza de transacciones } \\
\text { corrientes (en \% del pib) }\end{array}$ & 6.3 & 7.4 & $7.0 \mathrm{e}$ & $6.2 \mathrm{e}$ & 5.8 \\
\hline
\end{tabular}

Fuente: Banco Santander, 2015 
En relación con el crecimiento económico, los indicadores alemanes han prolongado sus buenos resultados en 2014 El Gobierno ha tomado medidas sociales como la implementación de un sueldo mínimo general, una rebaja de la edad legal de jubilación o incluso la atribución de subvenciones a madres de familia Por primera vez desde 1969, el presupuesto de 2015 prevé un reequilibrio de las finanzas públicas, objetivo que se logró en 2014, un año antes del plazo previsto Este presupuesto, que se eleva a 300 mil millones de euros, se centra en la inversión, la investigación, la educación, el sistema de jubilación, el empleo, la cooperación para el desarrollo y la revolución energética La política de déficit nulo sin embargo ha sido criticada por ciertos sectores, que ven en ella un freno al crecimiento y la inversión Parael Gobierno alemán, la ortodoxia presupuestaria es lo que asegura el bienestar de las futuras generaciones; $y$, considerando la inestabilidad mundial y el compromiso del país con diversas misiones militares y humanitarias, ser precavido es importante De hecho, el país sigue estando endeudado por sobre el límite de $60 \%$ del pib fijado por las reglas de la ue El objetivo es reducirlo a menos de $60 \%$ de aquía 2024 (Banco Santander, 2015)

A pesar de estos esfuerzos, persiste el reto de la integración de la antigua Alemania Oriental, donde el desempleo es muy alto, al igual que las desigualdades entre pobre y ricos, las más significativas de la zona euro (Banco Santander, 2015)

El mayor reto ante esta desigualdad en términos de empleo radica en el ingreso Si el ingreso mínimo es generoso, se comprime la distribución salarial desde abajo y se aumenta la tasa de desempleo entre los trabajadores no calificados. Esto ocurre en Alemania Oriental después de la unificación. Para conciliar la meta ética de asegurar empleo con la meta de asegurar ingreso, se requiere que el Estado benefactor ofrezca un ingreso mínimo mayor a quien trabaja que a quien permanece inactivo(Sinn, 2008)

El Estado benefactor asigna fondos a condición de que el beneficiario no trabaje a cambio de una remuneración El Estado, mediante estas ayudas, retira gente del mercado laboral para integrarla al sector público pero no como funcionario pagado, sino como un receptor de dinero que está impedido de trabajaren forma remunerada, pues si lo hace pierde el subsidio (Sinn, 2008)

El empleado alemán desea que su salario en el sector privado sea superior al salario de reemplazo que le paga el Estado Al mismotiempo, el empleador 
desea que el salario sea inferior a la productividad de ese trabajador Como resultado de ambas desigualdades, no existen empleos para aquellos ciudadanos cuya productividad laboral es inferior al salario de reemplazo ofrecido por el Estado Estas desigualdades explican lo que ocurre en el mercado laboral alemán respecto de los trabajadores de menor productividad: el subsidio de cesantía alemán es un destructor de empleos (Sinn, 2008)

Una comparación entre 1975 y 2005 hecha por Sinn (2008) muestra las tasas de desempleo para distintos niveles educacionales entre 1975 y 2005 No se observan grandes variaciones entre las personas con título universitario, pero entre aquellas que han completado sus programas de capacitación técnica, la tasa de desempleo casi se duplicó durante ese periodo, aunque sólo llegó a 7.4\% en 2005. Pero la situación para los trabajadores no calificados - que carecen de capacitación técnica — fue muy diferente, pues su tasa de desempleo aumentó en cerca de 18 puntos porcentuales durante esas tres décadas (Sinn, 2008)

En el caso alemán, en una primera etapa las fuerzas del mercado tendrían que haber hecho converger las cifras de productividad, dejando para una segunda etapa la convergencia de los salarios Este orden temporal era necesario debido al déficit inicial de Alemania Oriental en maquinaria e infraestructura moderna, y cubrir ese déficit tiene costos de transición que tendrían que ser financiados mediante un excedente de la productividad por sobre los salarios Sin embargo, sucedió todo lo contrario: los políticos decidieron aumentar primero los salarios y luego la productividad (Sinn, 2008)

El Estado benefactor comenzó a pagar salarios que fluctuaban entre $60 \%$ y $85 \%$ del nivel aplicado en Alemania Occidental En dichas circunstancias, la opción de pagar salarios bajos sencillamente deja de existir, porque el trabajador siempre puede dirigirse a una oficina del Gobierno para pedir apoyo, en lugar de trabajar a cambio de un salario bajo (Sinn, 2008)

Un factor adicional: donaciones Éstas hacen que se consuma más de lo que se produce. También, si otros países prestan o financian inversiones físicas dentro del territorio, hay más recursos para gastar En Alemania Oriental la producción propia (pib) alcanza sólo a 287,000 millones de los 416,000 millones de euros De la diferencia, 98,000 millones de euros corresponden a transferencias fiscales netas desde Alemania Occidental a Alemania Oriental, por la vía del presupuesto federal De esta forma sus habitantes pueden 
consumir mucho más de lo que producen (Sinn, 2008) Afortunadamente Alemania Occidental es un país rico: la donación equivale a 4 5\% de su pib, de modo que puede financiarla sin sufrir demasiado (Sinn, 2008).

A fin de disminuir la competencia, Schröder en la Agenda 2010 otorgó más dinero a quien trabaja y menos a quien permanece inactivo: los desempleados recibieron menos fondos Esto dio como resultado 400,000 nuevos empleos de baja remuneración, pero sin bajos salarios éstos no habrían sido creados (Sinn, 2008) Este mecanismo a corto plazo parece minar el valor porque lo disminuye en términos económicos (menos fondos para los desempleados), pero a largo plazo generó más valor (más puestos de empleo)

Hasta esta parte se analizó que con un salario alto, la desigualdad será mayor si los agentes económicos se concentran en estrategias de competitividad a corto plazo En este último caso, ante una visión de corto plazo resulta atractivo operar bajo el mecanismo de competencia, aunque esto conduzca a una pérdida mayor en el largo plazo Tal como se verá en el siguiente apartado

\section{Salarios y formas contractuales: sector automotriz mexicano}

La liberalización económica y la privatización iniciadas en México en la década de los ochenta del siglo pasado derivaron en la flexibilidad laboral (Canales, 2000; Contreras, 2000; Ibarra y González, 2010). Esto con el fin de quela entrada de capitales para inversión, debido a la transnacionalización de las actividades, no chocara con la rigidez de los contratos laborales mexicanos

El30denoviembre del2012 fue publicadaen elDiario Oficialdela Federación la reforma laboral, en la que se modificó la Ley Federal del Trabajo de 1970 con el argumento de que esta reforma elevaría la generación de empleos con la inclusión de jóvenes y mujeres al mercado formal de trabajo (Díaz, Ramírez y Salas, 2015)

El principal objetivo microeconómico de la flexibilidad laboral ha sido aumentar la eficiencia y la productividad por trabajador; sin embargo, este mecanismo no ha mejorado las condiciones del empleado mexicano A pesar de que el mercado laboral mexicano es institucionalmente flexible (Díaz, Ramírez y Salas, 2015)

De acuerdo con Díaz y otros (2015) la flexibilidad laboral en términos numéricos ha actuado como un mecanismo ahorrador de mano de obra que, 
acompañado de bajas tasas de crecimiento económico, ha promovido un entorno de austeridad en la generación de empleos formales, por lo que impide la inserción de nueva fuerza laboral en el mercado formal de trabajo, que se convierte en un remanente acumulativo que ha engrosado la tasa de desempleo Asimismo, se encuentra una bidireccionalidad positiva entre la tasa de desempleo y la flexibilidad laboral, por lo que la condición de desempleo puede ser un motivo por el cual los empleadores consiguen proteger la rentabilidad a través de contratos temporales (Díaz, Ramírez y Salas, 2015)

Diversos autores ya habían señalado que la deslocalización del empleo sólo conduciría a condiciones laborales cada vez más precarias (Castillo, 1989; Novick, et al , 1998; Yoguel, et al., 2001; Boyer y Neffa, 2004; Drolas, Montes Catóy Picchetti, 2005; Delfini y Cross, 2005). Ésteha sidoel caso de México.

La forma en que Delfini (2010) analiza la situación permite identificar los mecanismos que generan valor social y que hasta este momento suelen ser ignorados en materia laboral Este autor asumió a la historia del capitalismo como la historia de las formas que asume el control sobre el proceso de trabajo, poniendo énfasis en el análisis y la explicación de las formas de control que se despliegan en los espacios laborales, abordando el sector automotriz (Delfini, 2010; Gaudemar, 1991; Edwars, 1990; Friedman, 1977; Castillo Mendoza, 1991; Braverman, 1984; Hyman, 1981; Katz, 2000; Edwards, 2000; Sewel, 2001; Lahera, 2000, 2005)

Dentro de la primera posición descrita por Delfini (2010) se encuentran los análisis realizados por Andrew (1977). Este último define al control como no lineal, con diferentes direcciones y diversas formas, dependiendo, entre otras causas, de las posibles resistencias de los trabajadores y la competencia en el mercado

Andrew (1977) distingue dos estrategias de control: la primera es el "control directo" que se pone de manifiesto a través de las supervisiones directas, amenazas coercitivas y poca o nula responsabilidad de los trabajadores sobre el proceso de trabajo y sus productos La segunda comprende un grupo que el autor denomina "estrategias de autonomía responsable", que apunta a potenciar las iniciativas de los trabajadores en busca de la adaptación de éstos a situaciones cambiantes, a la vez que suponen un control de carácter ideológico que intenta conseguir la adhesión de los trabajadores al proceso productivo 
La empresa compra el tiempo total de uso de la fuerza de trabajo pero no su intensidad, ni el esfuerzo requerido Éstos son componentes de carácter endógeno, a partir de los cuales se lleva adelante el proceso de extracción de plusvalía En este sentido, la amenaza latente de perder el trabajo aparece como mecanismo disciplinario cuando no se lleva adelante el proceso de trabajo bajo los esfuerzos requeridos de manera endógena (Delfini, 2010; Bowles y Gintis, 1990) Este mecanismo disciplinario se debilita en entornos en los que el gobierno ofrece un seguro de desempleo, dando libertad al trabajador de renunciar a la empresa sin necesariamente contar con una nueva fuente de empleo El Estado actúa como protector del trabajador pero no del puesto que abandona, afectando a la productividad de la empresa y sus resultados

La existencia de mecanismos complementarios que se concentren en el puesto en lugar de sólo en el trabajador permite que la empresa ante tales circunstancias pueda con mayor prontitud sustituir al personal que abandona dicho puesto sin afectar los resultados que finalmente garantizan la existencia de los puestos de empleo que dicha organización ofrece

Es interesante notar que para Burawoy (1989) el capitalismo ya no necesita de métodos coactivos para ejercer su dominio, sino que el control se logra por medio de la interiorización de un individualismo competitivo, que se refuerza en el ámbito de la empresa por medio de dos mecanismos: los mercados internos de trabajo y la negociación colectiva (Delfini, 2010). En este caso, se remite a mecanismos alternos que generan valor social y que no sólo requieren de estrategias de competencia individuales, llamado en palabras de los autores "individualismo competitivo" (Burawoy, 1989; Delfini, 2010), sino también se requiere de estrategias de cooperación conducentes a la reorganización de los miembros de la empresa en grupos de colaboración Estos grupos internos facilitan la negociación y permiten un equilibrio entre la competencia y la cooperación interna, aumentando la productividad y la estabilidad laboral

Los procesos de tercerización de firmas, el trabajo en equipo, la polivalencia, la autonomía de los trabajadores e incremento de la participación de éstos en diferentes instancias, el desarrollo de la calidad y la capacidad de adaptación de los trabajadores se vinculan al cambio en las formas de ejercicio del control sobre el proceso de trabajo, puesto que con la introducción de estas 
transformaciones se obtiene el compromiso voluntario de los trabajadores conla empresa, resaltandolas ventajas del carácter cooperativo entre capital y trabajo (Delfini, 2010). Todoesto da lugar a la implicación de los trabajadores con los valores que determina la empresa en busca la autodisciplina, el autocontrol y la vigilancia entre los mismos trabajadores (Garrahan y Steward, 1992; Sewel y Wilkinson, 1992; Wright y Edwards, 1998; Pulignano, 2002; Lahera, 2005; Montes Cató y Pierbattisti, 2007)

El sistema capitalista requiere del equilibrio entre la generación de valor económico y social para su prolongación (González, 2012) De esta forma, cuando se generan nuevos mecanismos de competencia, éstos llevan aparejada la generación de nuevos mecanismos de cooperación Si este equilibrio no se sostiene, el sistema colapsa Para el caso de la empresa y sus vinculaciones internas, se considera que éstallegaría a su fin. La generación de mecanismos de competencia en el mercado laboral que facilitan la expansión de los capitales ha derivado en la existencia de nuevos mecanismos de cooperación que permiten la conservación de los puestos de empleo y reorientan las acciones hacia lageneración de valor social

La participación de los trabajadores en la empresa es clave para los nuevos modelos organizacionales, porque se aprovechan los conocimientos adquiridos en instancias de trabajo para ser transferidos a la empresa Estos modelos están centrados en la implantación de culturas empresariales que rompen con las prácticas tayloristas (Lahera, 2004)

Las estrategias empresariales para mayor control están ligadas a las transformaciones en la organización del proceso de trabajo Así, las innovaciones organizacionales, la utilización de nuevos mecanismos de regulación, la aplicación de nuevas tecnologías y la gestión de la participación aparecen como instrumentos utilizados por los gerentes para romper con el pasado y promover una nueva clase vinculada a la empresa (Linhart, 1997)

Estos cambios se han dado en el sector automotriz e implican la redefinición de las relaciones entre terminales y proveedoras, dando como resultado la conformaciónjerárquica del sectoren varios niveles. Así, la firma Terminal actúa como núcleo, convirtiéndose en una ensambladora y tercerizando parte de lo que constituía originalmente su producción (Yoguel, Novick y Marín, 2001; Varela, 2003; Delfini y Cross, 2005). 
Esta tercerización disminuye el costo de la producción de las autopartes, naciendo la diferenciación de los salarios entre los obreros de la armadora y los obreros de las empresas de autopartes, así como en algunos casos la pérdida del poder sindical al participar sindicatos diferentes entre las armadoras y las empresas de autopartes

La estrategia empresarial implica desde acciones coercitivas hasta las que consiguen sutilmente el consentimiento de los trabajadores en el proceso de producción. Delfini (2010) analizó diversos espacios de trabajo del sector automotriz, siendo la fuente de información los trabajadores de las empresas Los resultados de este investigador evidencian la filosofía de trabajo de las empresas, la polivalencia y la posibilidad de que los trabajadores ocupen diferentes posiciones, de acuerdo con los requerimientos de la producción y con la organización del trabajo determinada por la firma; muestra la forma en que se procesa el "saber estar social" requerido por las firmas a sus trabajadores. En otras palabras, implica la capacidad de adaptación, de comprensión y de interacción que requieren las firmas de sus asalariados; el sindicato ha dejado de ser un referente primordial, para quedar reducido a "recurso de última instancia" porque ya no representa una opción válida para canalizar inquietudes y ver reflejados los intereses del trabajador (Delfini, 2010).

Uno de los casos abordado por el investigador evidencia mejor quién absorbe el riesgo de las operaciones En el caso de la productora de mazos de cable existe una remuneración variable que se otorga de acuerdo con la calidad, siempre que no existan devoluciones de productos por parte de los clientes. Esto pone de manifiesto la presencia de la Terminal en el control sobre los trabajadores de la empresa de autopartes De esta manera, la posibilidad de incrementar el salario por parte de los trabajadores queda supeditada a la calidad determinada por la empresa núcleo porque se sabe dónde y cuándo se produjo el defecto (Delfini, 2010).

Es evidente que las estrategias empresariales han estado enfocadas en aumentar la competitividad y en disminuir los costos, mediante la intensificación del trabajo al mismo tiempo que el salario es disminuido, aun con la existencia de un salario mínimo Canalizan los esfuerzos de los trabajadores hacia estrategias de competencia que al mismo tiempo reflejan la orientación de la empresa a la que pertenecen Todo pareciera efectuarse bajo la estrategia de arrebatar valor, antes que compartirlo 
Diferentes estudios realizados sobre el comportamiento del salario en México concluyen que la desigualdad salarial creció desde mediados de los ochenta hasta la segunda mitad de los noventa Castro (2007) estudia el comportamiento de la desigualdad salarial centrándose en la desigualdad salarial urbana e intraurbana y el periodo de estudio se extiende hasta los primeros años de este siglo

Los principales resultados que Castro (2007) destaca son: la existencia de un crecimiento de la desigualdad salarial hasta 1997; a partir de esa fecha se observa una contracción La descomposición de la desigualdad indica que el crecimiento de la disparidad estuvo impulsada por incrementos en la desigualdad tanto intragrupo como entre grupos, y posterior a 1997 la desigualdad intragrupos desciende pero las diferencias entre grupos continúan aumentando, ello para el caso de la clasificación por sexo como por ciudades (Castro, 2007)

La principal fuente de disparidad tanto al interior de las ciudades mexicanas como entre ellas hasta el año 2002 fue el factor salario, mientras que el análisis de la disparidad salarial por áreas urbanas permite establecer que el aumento de la desigualdad salarial promedio entre ciudades no se encuentra en las diferencias de dotaciones de factores sino en las remuneraciones que los recursos consiguen en los distintos centros urbanos; además el comportamiento parece abarcar una clara connotación espacial por lo cual durante el periodo de estudio parece observarse una mayor desigualdad salarial en términos geográficos (Castro, 2007)

La descentralización de la determinación de los salarios ha inducido un comportamiento dinámico de la tasa de salarios, el cual resulta en una mayor inequidad salarial El aumento de la inequidad salarial ha sido observado por nivel educativo, categorías ocupacionales, industrias y género entre otros tipos de clasificación. Esto explica el hecho de que la mayoría de los estudios sobre el mercado de trabajo en México hayan estado más concentrados en estudiar el grado de inequidad salarial y tratar de explicar su comportamiento Los estudios sobre inequidad salarial concluyen que detrás de este fenómeno se encuentra el aumento de la tasa de retorno relativa de los niveles educativos altos Educación es, pues, la variable catalizadora de este proceso de desigualdad salarial (Islas-Camargo y Cortez, 2004) 
El mercado laboral mexicano no es un mercado único integrado, es un conjunto de mercados laborales de alguna manera interconectados Existen diferentes explicaciones acerca del aumento de la desigualdad salarial en México La primera, y más aceptada, es que el aumento de la demanda relativa por mano de obra calificada es el factor determinante detrás del aumento de la brecha salarial. Dentro de esta perspectiva es posible identificar diferentes argumentos sobre las variables y los mecanismos que han generado los cambios de la demanda relativa: industrialización conducida por las exportaciones, cambio tecnológico, e inversión extranjera directa La segunda visión adopta una perspectiva institucional Ésta argumenta que la liberalización continua del mercado laboral mexicano ha causado la erosión de algunas instituciones del mercado laboral que ha resultado en el aumento de la desigualdad salarial (Islas-Camargo y Cortez, 2004)

Es posible que el aumento de la brecha salarial entre mano de obra calificada y no calificada se deba a una mayor demanda relativa de la primera que resulta del aumento de bienes de capital importado causado, a su vez, por la liberalización del comercio internacional (Cañonero y Werner, 2002) Esta mayor demanda relativa se debe a dos hechos Primero, capital y mano de obra calificada son sustitutos. Segundo, mano de obra calificada es sector-específico. Aún más, según Cañonero y Werner, la hipótesis de StolperSamuelson acerca del ajuste de los precios relativos de los factores de producción debe ser considerada como una predicción de largo plazo, mas no de corto o mediano plazo (Islas-Camargo y Cortez, 2004)

Campos (1995) explica que, en 18 años, México disminuyó las condiciones laborales de los asalariados del país por tres medios: a) imposición de topes salariales casi siempre por debajo de la inflación; b) debilitando las bases fundamentales del derecho sindical en México, el derecho de huelga, el de sindicalización y el de contratación colectiva, y c) imponiendo nuevas y más intensivas formas de trabajo a quienes han logrado conservar su fuente de trabajo, junto al desempleo y la amenaza de la reorganización tecnológica de la producción (Campos, 1995)

El estudio realizado por Castellanos (2012) evidencia grandes disparidades en los salarios de la industria automotriz instalada en México Esto se muestra al desglosarlos entre ensambladoras y fábricas de autopartes, así como entre obreros y empleados de la administración Los resultados encontrados 
muestran un promedio general de 45 salarios mínimos por trabajador de esta industria Al dividir el salario entre empleados de armadoras y de autopartes, se obtuvieron 89 veces para la primera y 41 para la segunda; al realizar la misma operación entre empleados administrativos y obreros, se obtuvo en armadoras 171 y 62 respectivamente; en autopartes 99 y 33 También se compararon los salarios que se pagaron en 2003 y 2008, encontrando que el incremento en los salarios fue menor que todos los demás indicadores, destacando la diferencia con el valor de la producción de la industria automotriz, que en dichos años creció en $49 \%$ y los salarios solamente en 18\% (Castellanos, 2012)

Entre los principales resultados se observa que (Castellanos, 2012): 1) el aumento del salario mínimo de 2003 a 2008 fue de 20 5\%; 2) el valor de la producción automotriz aumentó en $49 \%$; 3 ) las unidades producidas se incrementaron $37.5 \%$; 4) la inflación de junio de 2003 a junio e 2008 se incrementó $23 \%$. Esto significa que la inflación fue mayor que el aumento a las remuneraciones totales y al salario mínimo

Cuando el salario no corresponde al necesario para que el trabajador lleve una vida digna (aun cuando simple), al mismo tiempo que el producto en otra región es vendido a un precio elevado, se considera que se ha arrebatado el valor, social antes que el económico, generado por los trabajadores Nótese que se señala al necesario, que no es sinónimo de más alto pero que tampoco justifica una disminución en el mismo. De esta misma forma, los agentes económicos que operan en una localidad deben concentrarse en promover todo aquello que dignifique la vida de sus habitantes en concordancia con su propio contextohistórico

Cuando el salario tiende a bajar, debido a la estrategia de las empresas, se arrebata el valor social de la localidad que ofrece mano de obra barata y demás recursos para la producción transnacional

\section{Conclusiones}

Las actividades económicas que generan valor social son aquellas que proporcionan instrumentos y nuevas formas para aumentar la calidad de vida de la población trabajadora Quienes, a su vez, se incentivan para trabajar 
más en pro de la generación de valor económico A esto se añade el efecto multiplicador

El salario es visto por muchos como el principal medio para elevar la calidad de vida de la población y contribuir al Estado de bienestar; sin embargo, éste por sí mismo no es una garantía Se requiere de estrategias complementarias que permitan un equilibrio entre la generación de valor social y económico derivado de la articulación de los agentes económicos con la población en general El caso de Alemania muestra la forma en que el mecanismo de competencia es el que determina el freno al crecimiento, debido a que se agota el valor social en aras de obtener lo máximo posible del valor económico Aun cuando se tuviese la impresión de que este mecanismo es usado con el fin de garantizar un Estado benefactor.

En el caso de México, los salarios en la industria automotriz son altos comparados con la industria en general y con los otros sectores económicos en México Sin embargo, los salarios de las ensambladoras duplican al correspondiente a los trabajadores de autopartes Las asimetrías en los ingresos del sector contribuyen a la inflación, fomentan la precariedad laboral e incentivan eloutsourcing

Las donaciones de Alemania Occidental a Alemania Oriental generan el mismo efecto que los salarios altos ofrecidos por las transnacionales del sector automotriz en México Incentivan el consumo sin con ello garantizar un incentivo para la producción La población con mayor ingreso pero con menor productividad consume más de lo que se produce, provocando mayor pobreza y desempleo a largo plazo

\section{Referencias bibliográficas}

Banco Santander (2015) Alemania, política y economía Santander Trade Portal Fecha de consulta: 20 septiembre 2015 Link https://es santandertrade com/analizarmercados/alemania/politica-y-economia

Banxico (2005) Principales indicadores salariales en México México: Banco de México

Bowles, S, y Gintis, H (1990) "The revenge of Homo Economicus: Contested Exchange and the Revival of Political Economy", Journal of Economic Perspectives, 7(1)

Boyer, R, y Neffa, J (2004) La economía argentina y su crisis (1976-2001): Visiones institucionalistas y regulacionistas. Buenos Aires: Miño y Dávila 
Braverman, H (1984) Trabajo y capital monopolista. La degradación del trabajo en el siglo $x x$ México: Nuestro Tiempo

Burawoy, M (1989) El consentimiento en la producción. Los cambios del proceso productivo en el capitalismo monopolista Madrid: Ministerio de Trabajo y Seguridad Social

Campos, L (1995) “El ciclo largo de la caída salarial en México”, Problemas del Desarrollo, 26(102)

Canales, A. (2000). "Migración internacional y flexibilidad laboral enel contexto del tlcan”, Revista Mexicana de Sociología, 3(28)

Cañonero y Werner (2002) "Salarios relativos y liberalización del comercio exterior en México”, ElTrimestre Económico, vol lxix(1), núm 273,pp 123-142

Castellanos, J(2012) "Sueldos y salarios de la industria automotriz en México en 2003 y 2008: Aguascalientes, Estado de México, Puebla, Chihuahua y Coahuila de Zaragoza", Memoria del xvii Congreso Internacional en Contaduría, Administración e Informática México: unam-fca

Castillo, C (1991) "Estudio introductorio", en Gaudemar, J P, El orden y la producción. Nacimiento y formas de la disciplina de fábrica. Madrid: Trotta

Castillo, J (1989) "La división del trabajo entre empresas", Sociología del Trabajo, núm 5,invierno Madrid

Castro, David (2007) “Disparidad salarial urbana en México, 1992-2002”, Estudios Sociales (Hermosillo, Son.), 15(29): 117-153

Contreras, Ó. (2000). "Los estudios acerca delaflexibilidad laboral en México: Algunas observaciones críticas", Estudios Sociológicos, pp 727-735

Delfini, M. (2010). "Prácticas y estrategias empresarias para la reproducción de la dominación en los espacios de trabajo: Formas de control obrero en el sector automotriz", Trabajo y Sociedad, núm 14

Delfini, M., y Cross, M. C. (2005). "La construcción de hegemonía en las empresas. El caso de una firma en el sector automotriz", en Fernández, Arturo, Estado y relaciones laborales: Transformaciones y perspectivas. Buenos Aires: Prometeo

Díaz, E., Ramírez, E., y Salas, E. (2015). “La ley de Okun y la flexibilidad laboral en México: Un análisis de cointegración”, Contaduría y Administración, 60(3)

Drolas, A, Montes, J, y Picchetti, V(2005) "Las relaciones de poder en los espacios de trabajo", en Fernández, A (Comp), Estado y relaciones laborales Buenos Aires: Prometeo

Edwards, P(1990) El conflicto en el trabajo. Madrid: Ministerio de Trabajo y Seguridad Social deEspaña

(2000) "Discipline”, en Bach, S, y Sisson, K (Eds ), Personnel management. Oxford, Reino Unido: Basil Blackwell 
Feliciano, Z (1995) Essays on Economic Policy and Labor Market Outcomes: Trade Liberalization, Minimum Wages and Migration. The Case of Mexico Tesis doctoral, Harvard University

Friedman, A (1977) Industry and labour. Class struggle at work and monopoly capitalism. Londres: Macmillan Press

Garrahan, P , y Steward, P (1992) The Nissan enigma. Londres: Casell

Gaudemar, J P(1991) El orden y la producción. Nacimiento y formas de la disciplina de fábrica Madrid:Trotta

Gobierno de la República (s/f) Constitución Política de los Estados Unidos Mexicanos

González, T (2015a) "Fragilidade nas redes internacionais de cooperação empresarial", Administração Faces Journal, 11(3), año 2012

(2015b) "Rasgos de la responsabilidad social en las empresas española, argentina y mexicana participantes en Al-Invest”, Revista Universitaria Europea, 1(22)

Gray, G(2015) "La última legua de erradicación de la pobreza", Humanum Consultado el 26 de octubre Link http://www revistahumanum org/blog/la-ultimalegua-de-erradicacion-de-la-pobreza/\# vi5ewpud1vm facebook

Hyman, R (1981 [1975]) Relaciones industriales. Una introducción marxista Madrid: Blume Ediciones

Ibarra, M. A., y González, L. A. (2010). “La flexibilidad laboral como estrategia de competitividad y sus efectos sobre la economía, la empresa y el mercado de trabajo", Contaduría y Administración, núm 231, pp 33-52

Islas-Camargo, A, y Cortez, W(2004) "Convergencia salarial entre las principales ciudades mexicanas: Un análisis de cointegración”, EconoQuantum, 1(1): 25-47

Katz, C (2000) "La teoría del control patronal: Balance de una discusión”, Época, revista argentina de economía política, año 2, núm 2 Buenos Aires

Lahera, A (2000) "La emergencia de nuevos modelos productivos: La participación de los trabajadores y la fabricación del consentimiento en la producción”, Revista de Dialectología y Tradiciones Populares, tomo 1v, cuaderno segundo

(2004) "La participación de los trabajadores en la calidad total: Nuevos dispositivos disciplinarios de la organización del trabajo", Revista Española de Investigaciones Sociológicas, núm 106

— (2005) "Conquistando los corazones y las almas de los trabajadores: La participación de los trabajadores en la calidad total como nuevo dispositivo disciplinario”, en Castillo, Juan José, El trabajo recobrado. Buenos Aires: Miño y Dávila

Linhart, D (1997) La modernización de las empresas Buenos Aires: Asociación Trabajo y Sociedad 
Ministerio de Trabajo e Inmigración (2009) La política de subvenciones entre el fomento de la competitividad y la estabilización del empleo", Actualidad Internacional Sociolaboral, núm 125

Montes Cató, J, y Pierbattisti, D (2007) "Relaciones de poder y disciplinamiento en los espacios de trabajo Un estudio sobre la dominación en empresas de telecomunicaciones", Estudios del Trabajo, núm 33

Novik, M, Miravalles, M, y Senén González, C (1998) Estudios empíricos de sectores productivos en los diferentes países: Vinculaciones interfirmas y competencias laborales en la Argentina, los casos de la industria automotriz y las telecomunicaciones oit Centro Interamericano de Investigación y Documentación sobre Formación Profesional Montevideo: Cinterfor

Pulignano, V(2002) "Dynamic forms of control at work: A research note on integrated supply chains in the motor industry in southern Italy", Work, Employment and Society. Londres: bsa Publications, volume 16

Sewel, L G (2001) "What goes around comes around Inventing a mythology of teamwork and empowerment", Journal of Applied Behavioral Sciences, 37(1)

Sewel, L G, y Wilkinson, B (1992) "Someone to watch over me: Surveillance, discipline, and just-in-time labor process", Sociology, 26(2)

Sinn, H W (2008) "Mercado laboral: Lecciones del Estado de bienestar alemán”, Estudios Públicos, núm 109, pp 5-36

Varela, L (2003) "El sector automotriz argentino Estudio sobre la cadena productiva local", Realidad Económica, núm 196

Wright, M, y Edwards, $\mathrm{P}(1998)$ Does team working work, and if so, Why? A case

Yoguel, G, Novick, M, y Marin, A (2001) "Estilos de vinculación, procesos de innovación y tecnología de gestión social", Redes, núm 17 